\title{
Preparation, Characterization, and Bioactivity of Chitosan Microspheres Containing Basic Fibroblast Growth Factor
}

\author{
Bo Lv, Yue Wang, and Wei Chen \\ Department of Orthopaedics, Sichuan Academy of Medical Science, Sichuan Provincial People's Hospital, Chengdu 610041, China \\ Correspondence should be addressed to Yue Wang; wangyue0218@yeah.net
}

Received 19 May 2014; Accepted 18 July 2014; Published 14 October 2014

Academic Editor: Chunyi Zhi

Copyright (C) 2014 Bo Lv et al. This is an open access article distributed under the Creative Commons Attribution License, which permits unrestricted use, distribution, and reproduction in any medium, provided the original work is properly cited.

\begin{abstract}
The aim of this study is to evaluate, prepare, and characterize bioactivity of chitosan microspheres loaded with bFGF for providing sustained release of bFGF. Porous chitosan microspheres were prepared by freeze-drying process based on the interaction between chitosan and tripolyphosphate (TPP). The bFGF-loaded chitosan microspheres were well interconnected and have a narrow size distribution, spherical shape, and positive surface charges. The bFGF-loading capacity and encapsulation efficiency were $7.57 \mathrm{mg} / \mathrm{g}$ and $95.1 \%$, respectively. Results of in vitro release showed that the extent of release was $82.1 \%$ at Day 25. Schwann cells were used as an in vitro model for cell response to bFGF and bFGF-loaded chitosan microspheres. Results indicated that the number, cell viability, and percentage of cells $\mathrm{G} 2 / \mathrm{M}+\mathrm{S}$ phase in the bFGF groups are higher than those in the bFGF-loaded chitosan microspheres groups before culturing for 2 days. However, the number, cell viability, and percent of cells G2/M+S phase in the bFGF-loaded chitosan microspheres groups are significantly higher than those in the bFGF groups after culture for 4 and 8 days. These findings indicated that bFGF-loaded chitosan microspheres may help to decrease the release of bFGF and provide a suitable three-dimensional environment for cell growth and proliferation.
\end{abstract}

\section{Introduction}

Fibroblast growth factors (FGFs) constitute a family of heparin binding polypeptides, which are involved in the regulation of biological responses, such as growth, differentiation, and angiogenesis [1]. Basic fibroblast growth factor (bFGF) is a member of the FGF family that comprises nine members. This FGF prototype has pleiotropic effects in different cell and organ systems. bFGF is a potent angiogenic molecule that in vivo and in vitro stimulates smooth muscle cell growth, wound healing, and tissue repair $[2,3]$. In addition, bFGF may stimulate hematopoiesis and may play an important role in the differentiation and/or function of nervous system, eye, and skeleton [4]. However, the bioactivity of bFGF cannot always be expected when it is injected into the body by soluble form, because of its short duration of retention at wound sites and short half-life caused by susceptibility to enzymatic and thermal degradation in vivo [5]. Poor stability is considered to be one of the major factors that limit pharmacological applications of proteins, generating problems in formulation, administration, and storage. So, an effective delivery system of bFGF is required to overcome the shortcoming of bFGF application in a clinical setting.

Recently, a number of strategies and delivery methods have been designed and evaluated for the reportedly poor thermal stability and a relatively short in vivo half-life $[6,7]$. One of the approaches for enhanced thermostabilization of proteins is to alter the microenvironment using additives. Additives may modify the solvent structure such as sugar, arginine, heparin, and so forth, which have been tried with considerable success for understanding the mechanism of thermal inactivation in some growth factors [5]. Another option to enhance in vitro and in vivo efficacy of growth factors is to incorporate them into polymeric biomaterials, like scaffold, microsphere, gel, and so forth, which may maintain their stability and control their release kinetics. The use of microsphere-based therapy allows drug release to be carefully tailored to the specific treatment site through the choice and formulation of various drug-polymer combinations. The total dose of medication and the kinetics of release are the 
variables, which can be manipulated to achieve the desired result. Using innovative microencapsulation technologies, and by varying the copolymer ratio, molecular weight of the polymer, and so forth, microspheres can be developed into an optimal drug delivery system which will provide the desired release profile. Microsphere-based systems may increase the life span of active constituents and control the release of bioactive agents [8-10].

Chitosan is a biodegradable natural polymer with potential for pharmaceutical applications for its biocompatibility, high charge density, nontoxicity, and mucoadhesion. It not only improves the dissolution of poorly soluble drugs but also exerts a significant effect on fat metabolism in the body $[11,12]$. Thus, chitosan possesses suitable properties as a carrier for microsphere drug delivery. Chitosan microspheres are used to provide controlled release of many drugs and to improve the bioavailability of degradable substances such as protein or enhance the uptake of hydrophilic substances across the epithelial layers. Chitosan has also been used as a potential carrier for prolonged delivery of drugs, macromolecules, and targeted drug delivery. Magnetic chitosan microspheres used in targeted drug delivery are expected to be retained at the target site capillaries under the influence of an external magnetic field [11]. Various devices, including microspheres, scaffolds, and hydrogels, have been used to bind and release angiogenic growth factors, such as vascular endothelial growth factor (VEGF), acidic and basic fibroblast growth factor (aFGF and bFGF), and platelet-derived growth factor (PDGF) $[6,7]$. These reports will help to understand the potential application of microspheres and deliver growth factors in tissue repair engineering. However, further extensive research will be carried out to exploit chitosan as a growth factor carrier to attain the desirable results. Thus, bFGF was chosen to incorporate into the chitosan microspheres based on the emulsion cross-linking method. The morphology, particle size and distribution, bFGF-loading capacity, encapsulation efficiency, and in vitro release rates were analyzed using electron microscopy, laser particle size analyzer, potentiometric analyzer, and ELISA methods. In addition, the bioactivity of released bFGF was evaluated by measuring ability of the released bFGF to stimulate proliferation of Schwann cell.

\section{Materials and Methods}

2.1. Chemicals and Materials. Chitosan, sodium tripolyphosphate, liquid paraffin, sorbitan monopalmitate 80 , and so forth were obtained from Chengdu KeLong Chemicals (Chengdu, China). Human bFGF was supplied by PrimeGene (Shanghai PrimeGene Bio-Tech Co., Ltd., Shanghai, China). Antibodies used in the present work were purchased from Beijing Zhongshan Golden Bridge Biotechnology Co., Beijing, China. Dulbecco's modified essential medium (DMEM) and fetal bovine serum (FBS) were purchased from Gibco Invitrogen Corporation (Carlsbad, CA, USA). All other solvents used in this study are of analytical grade.

2.2. Preparation of bFGF-Loaded Chitosan Microspheres. Chitosan solutions with concentrations of 1.2 and $1.5 \%(\mathrm{w} / \mathrm{v})$

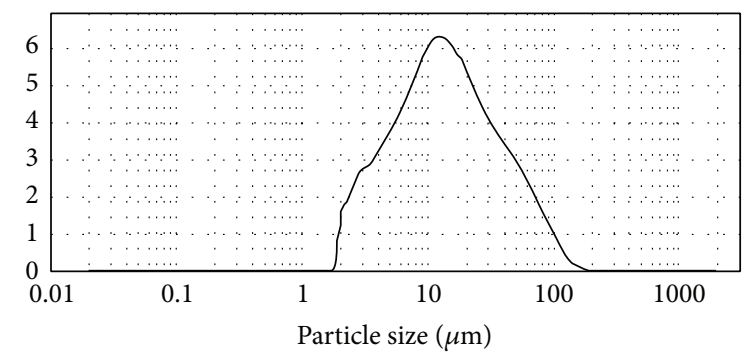

FIGURE 1: Particle size of bFGF-loaded chitosan microspheres.

were prepared by dissolution of chitosan flakes in $0.35 \mathrm{M}$ acetic acid and were filtered through $0.45 \mu \mathrm{m}$ membrane filter (Millipore) for removing any nondissolved residue particles. bFGF-loaded chitosan microspheres were prepared via freeze-drying method which was described as follows. Firstly, the concentrations of chitosan (1.2\% and $1.5 \%)$ and sodium tripolyphosphate $(0.6 \%$ and $1 \%)$ were selected based on the particle size and distribution of microspheres, and the experiments were designed as four groups. Reports from particle size analysis showed the concentrations of chitosan and sodium tripolyphosphate use $1.5 \%$ and $0.6 \%$ as optimized formulation for the preparation of microspheres. The particle size is about $21.554 \mu \mathrm{m}$ with a single peak, and the sizes in the other three groups show bimodal shape (Figure 1). Second, the bFGF-loaded chitosan microspheres were prepared based on the optimized formulation. The bFGF solution $(1 \mathrm{mg} / \mathrm{mL}$, $0.15 \mathrm{~mL}$ ) was mixed with chitosan solution $(1.5 \%, 1 \mathrm{~mL})$, and removed air bubbles in the solution using ultrasonic in the bath. The sodium tripolyphosphate $(0.6 \%, 0.4 \mathrm{~mL})$ was added to the above mixture, and then the mixtures were stirred at $500 \mathrm{rpm}$ for $60 \mathrm{~min}$. The chitosan-bFGF solution was added dropwise to liquid paraffin $(6.18 \mathrm{~mL}$, containing $0.18 \mathrm{~mL}$ SP80 ) stirring at $500 \mathrm{rpm}$ for $60 \mathrm{~min}$ and emulsified stirring at $500 \mathrm{rpm}$ for $48 \mathrm{~h}$ at $37^{\circ} \mathrm{C}$. The samples were centrifuged at $2,000 \mathrm{rpm}$ for $5 \mathrm{~min}$ at $4^{\circ} \mathrm{C}$, and the precipitate was washed several times with petroleum ether. The precipitates were then freeze-dried for $24 \mathrm{~h}$ at room temperature, and the powder was collected and stored at $4^{\circ} \mathrm{C}$ for further analysis.

2.3. Analysis of Particle Size and Morphology. The particle size and distribution of bFGF-loaded chitosan microspheres powder were analyzed using laser particle size and zeta potential analyzer. The morphology of microspheres powder was observed by scanning electron microscopy after spraying.

2.4. Analysis of bFGF-Loading and Entrapment Efficiency of Microsphere. The bFGF-loading capacity and encapsulation efficiency were carried out according to the instructions of ELISA kit. The standard equation was calculated based on the absorbance values of microspheres at $450 \mathrm{~nm}$. The bFGFloaded chitosan microspheres powder $(15 \mathrm{mg})$ was placed in the container containing PBS buffer solution ( $\mathrm{pH}$ 7.4, $50 \mathrm{~mL}$ ) and filtrated using filter membrane $(0.45 \mu \mathrm{m})$. The bFGFloading capacity and encapsulation efficiency were calculated 
based on the absorbance values in the washing liquid. The bFGF-loading capacity and encapsulation efficiency were calculated for each treatment using the following equations: bFGF-loading capacity $=$ (drug loading dosage - drug amount in the wash solution)/total amount in the microspheres; the encapsulation efficiency $(\%)=$ (drug loading dosage - drug amount in the wash solution)*100\%/total amount in the microspheres. The experiments were arranged in a completely randomized design with three replicates per treatment.

2.5. In Vitro bFGF Release Studies. Sixty milligrams of bFGFloaded chitosan microspheres was placed in the dialysis bag and suspended in $60 \mathrm{~mL}$ of PBS buffer ( $\mathrm{pH} 7.4$ ) as a medium at $37^{\circ} \mathrm{C}$ with mild agitation $(100 \mathrm{rpm})$. At several intervals, the supernatants were collected by centrifugation, and the amount of the released bFGF was determined by using ELISA method. The cumulative release rate was calculated for each treatment using the following equation: cumulative release rate $(\%)=W 1 / W 2 \times 100 \%$, where $W 1$ is bFGF amount in the wash solution and $W 2$ is the bFGF amount in the microspheres. The burst release rate is expressed as the cumulative release rate in the first day. All release studies were repeated three times and the results were reported as average values.

\subsection{Effects of bFGF-Loaded Chitosan Microspheres on the Cell} Number, Cell Viability, and Cell Phase. The experiments were separated into two groups. One group was allowed to culture with Dulbecco's modified essential medium (DMEM) containing $10 \%$ fetal bovine serum (FBS) plus $50 \mathrm{ng} / \mathrm{mL}$ bFGF as control. Another group was cultured with DMEM containing $10 \%$ FBS plus $50 \mathrm{ng} / \mathrm{mL}$ bFGF of microspheres. Widely used Schwann cells were chosen as in vitro experiment system. The Schwann cells were plated in 24-well plates at $1.0 \times$ $10^{4}$ cells $/ \mathrm{mL}$. The cells in two groups were collected at Days $1,2,3,4,6$, and 8 , respectively. The cell number was counted using cell counting plate. The cell viability was followed by MTT assay. In brief, the tetrazolium salt $(2 \mathrm{mg} / \mathrm{mL}$, $50 \mu \mathrm{L}$ ) was added to the wells before collection, and the cells were cultured for $4 \mathrm{~h}$. Then, the dimethyl sulfoxide $(150 \mu \mathrm{L})$ was added in the wells and stirred for $10 \mathrm{~min}$. The absorbance values in the wells were measured at $490 \mathrm{~nm}$ using a microplate reader (Bio-Tek. Instruments, Winooski, Vermont, USA). After culture, the cells were collected at Days 2 , 4, and 8 , and the cell phases were measured using flow cytometry. Then, the cell percentages of $\mathrm{G} 2 / \mathrm{M}+\mathrm{S}$ phase were calculated based on the analysis results. All release studies were repeated three times and the results were reported as average values.

2.7. Statistical Analysis. All treatments were arranged in a completely randomized design with three replicates. Data were expressed as mean \pm SD. Statistical significance was evaluated with Student's $t$-test, and differences were considered significant if $P$ values were less than 0.05 .

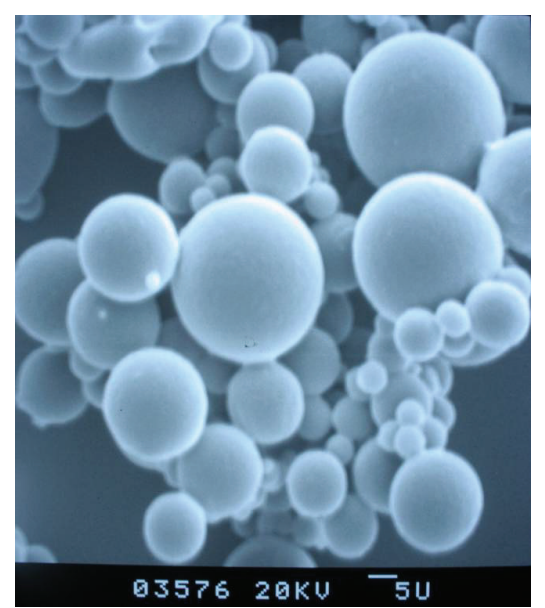

FIGURE 2: Structural properties of bFGF-loaded chitosan microspheres measured by scanning electron microscopy (SEM, ×4000).

\section{Results}

3.1. Morphology of bFGF-Loaded Chitosan Microspheres. As shown in Figure 1, the average diameter of $35.335 \mu \mathrm{m}$ of bFGF-loaded chitosan microspheres was observed using laser particle size and potential analyzer. Its size showed relative uniform distribution. As shown in Figure 2, the bFGF-loaded chitosan microspheres shown have interconnected structure and morphology, which have round smooth surface, no obvious pores, and good dispersity based on the results of scanning electron microscopy.

3.2. The bFGF-Loading Capacity, Encapsulation Efficiency, and In Vitro bFGF Release Studies of bFGF-Loaded Chitosan Microspheres. After measure and calculation, the concentration of bFGF in the washing solution was approximately $2.43 \mu \mathrm{g} / \mathrm{mL}$, and the total amount of bFGF was approximately $7.29 \mu \mathrm{g}$. Based on the calculated equations, the bFGF-loading capacity and encapsulation efficiency were $7.57 \mathrm{mg} / \mathrm{g}$ and $95.1 \%$, respectively. bFGF release from the bFGF-loaded chitosan microspheres started with an initial burst effect and continued with a controlled and steady release period. Total release of bFGF from the bFGF-loaded chitosan microspheres lasted as long as 24 days. At Day 1, bFGF was released by $180.8 \mathrm{ng} / \mathrm{mL}$, and the burst release rate in the first day was $18.1 \%$. The concentrations of bFGF reached $820.5 \mathrm{ng} / \mathrm{mL}$ from Day 2 to Day 24, and the average increase concentration was $53.3 \pm 12.8 \mathrm{ng} / \mathrm{mL}$ every two days. The cumulative release rate was $82.1 \%$ from Day 2 to Day 24 .

\subsection{Effects of bFGF and bFGF-Loaded Chitosan Microspheres} on the Cell Number. As shown in Figure 4, the cell number in themicrospheres groups at different cultured times shows significant difference compared to that of the control groups. The cell number in the microspheres groups is less by about $35.5 \%$ and $36.4 \%$ at Days 1 and 2 compared with the control groups. At Days 3 and 4, the number in the microspheres groups showed no significant difference compared with the control groups, respectively. The values in the microspheres 
groups are significantly higher than the control by $25.8 \%$ and $22.4 \%$ at Days 6 and 8, respectively.

\subsection{Effects of bFGF and bFGF-Loaded Chitosan Microspheres} on the Cell Viability. The effects of bFGF and bFGF-loaded chitosan microspheres on the cell viability are shown in Figure 5. The cell viability in the microspheres groups decreased by $26.8 \%$ and $36.2 \%$, respectively, compared with the control after 1 and 2 days of culture. After 3 and 4 days, the microspheres groups experienced no difference compared with the control groups, respectively. The cell viability of the microspheres groups was $33.1 \%$ and $22.3 \%$ higher than the control groups after 5 and 6 days, respectively.

3.5. Effects of bFGF and bFGF-Loaded Chitosan Microspheres on the Cell Phases. The effects of bFGF and bFGF-loaded chitosan microspheres on the percentages of $\mathrm{G} 2 / \mathrm{M}+\mathrm{S}$ phase were shown in Figure 6. As shown in Figure 6, the cell percentages of $\mathrm{G} 2 / \mathrm{M}+\mathrm{S}$ phase showed significant different after culturing for 2, 4 and 8 days between microspheres and control group. At Day 2, the percentages in the microspheres groups were significantly less by $42.1 \%$ compared to the control groups. However, the percentages in the microspheres groups were significantly higher by $51.8 \%$ and $97.4 \%$ compared with the control groups at Days 4 and 8, respectively.

\section{Discussion}

A multifunctional role of bFGF is suggested by many different receptor phenotypes expressed in various cell types. bFGF stimulates the growth of fibroblasts, myoblasts, osteoblasts, neuronal cells, endothelial cells, keratinocytes, chondrocytes, and many other cell types. It is assumed that bFGF will be of potential interest as a factor promoting wound healing and revascularization of tissues. Animal experiments with bFGF have shown that it promotes endosteal, but not periosteal, bone formation and bFGF may thus be a potential agent for treating osteoporosis, which may increase bone mass without causing outward deformation of the skeletal bones [1-3]. However, bFGF is a very unstable protein. At alkaline $\mathrm{pH}$, covalent aggregation of bFGF for thiol-disulfide exchange or $\beta$-elimination was observed. Under acidic conditions, degradation products of bFGF caused by peptide bond hydrolysis deamidation were generated. In addition, bFGF has a very short half-life in vivo at physiological $\mathrm{pH}$ and temperature [5], and this limits its therapeutic value for frequent administration. Thus, the sustained-release formulations will help to maintain the bFGF activity and/or improve the stability of bFGF and to prolong its effects in vivo. Chitosan is a partially deacetylated polymer obtained from the alkaline deacetylation of chitin, which is a glucosebased, unbranched polysaccharide. Chitosan exhibits a variety of physicochemical and biological properties resulting in numerous applications in fields. In addition to its lack of toxicity and allergenicity, its biocompatibility, biodegradability, and bioactivity make it a very attractive substance for diverse applications as a biomaterial in the pharmaceutical and medical fields $[13,14]$. Chitosan microspheres are used to provide controlled release of many drugs, such as insulin, antibiotics, growth factors, and so forth, which improve the bioavailability of degradable substances such as protein or enhance the uptake of hydrophilic substances across the epithelial layers [11]. The preparation methods of chitosan microspheres mainly include phase separation, ion-induced emulsion crosslinking, spray drying, and so forth [12]. In the present study, the emulsion crosslinking method was selected to prepare bFGF-loaded chitosan microspheres for the unstability of bFGF to heat and organic reagents. The preparation of microspheres may be affected by a variety of factors, like chitosan concentration, crosslinking agent, emulsion temperature, stirring speed, and so forth. Shu and Zhu reports indicated that microspheres have a good appearance of the sphere using crosslinker of sulfate salt, citrate salt, and TPP, and the microspheres have better mechanical properties, swelling resistance, and drug release properties using TPP as crosslinker [15]. TPP have some advantages, such as mild reaction conditions, simply operation, and little effects on bFGF activity. Thus, the present study selected TPP as a crosslinking agent for the preparation of microspheres. The present results showed that bFGF-loaded chitosan microspheres have a good appearance of the sphere, smooth surface, and good dispersion using 1.5\% of chitosan concentration and $0.6 \%$ of TPP (Figure 1). In addition, our results also showed that microspheres are easily dispersed and unevenly distributed with the increasing concentrations of chitosan and viscosity of chitosan solution (data not shown). The particle sizes are relative to stirring speed except for other parameters. The particle size gradually decreased with the increasing stirring speed, and the higher constant stirring helped to form a uniform particle size distribution of microspheres. Thus, the $100 \mathrm{rpm}$ of stirring speed was selected during the preparation process of microspheres. The microspheres are easily stuck together and are difficult to disperse for the water-in-oil system of chitosan microspheres. Thus, the higher temperatures help the evaporation of water, and the experiment temperature was selected at $37^{\circ} \mathrm{C}$ for reaction temperature. The Span-80 was selected as emulsifier in this study, which may form a protective layer on the surface of microspheres to prevent the adhesive interaction of microspheres. The results of Zeng et al. [16] showed that the particle size decrease and even distribution are observed with the increasing emulsifier concentration. However, the excessive emulsifier will be difficult for elution and will result in producing certain toxicity. Thus, the 3\% Span-80 was selected as the present experiment condition.

Drug loading capacity and encapsulation efficiency are important indicators of quality to drug-loaded microspheres, which were affected series of factors. The most important factor is the preparation process of microspheres $[11,12]$. The present study suggested that the bFGF-loadingcapacity and encapsulation efficiency reach $7.57 \mathrm{mg} / \mathrm{g}$ and $95.1 \%$ (Figure 3), respectively, which showed that the preparation process of bFGF-loaded chitosan microspheres is effective and feasible using emulsifying crosslinked method. Burst-release is the rapid release of drug from the formulation occurring in the initial $24 \mathrm{~h}$ of elution. There are generally burst release phenomena to biodegradable microspheres for protein and/or 


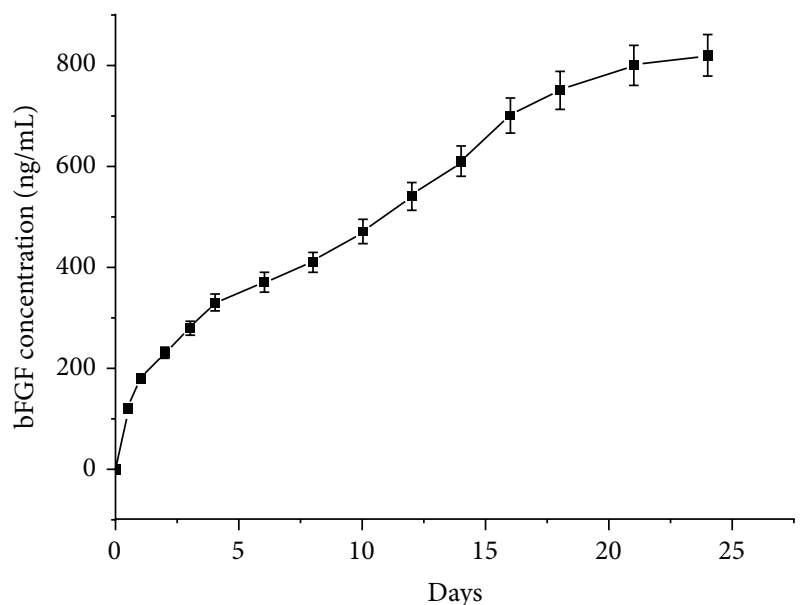

(a)

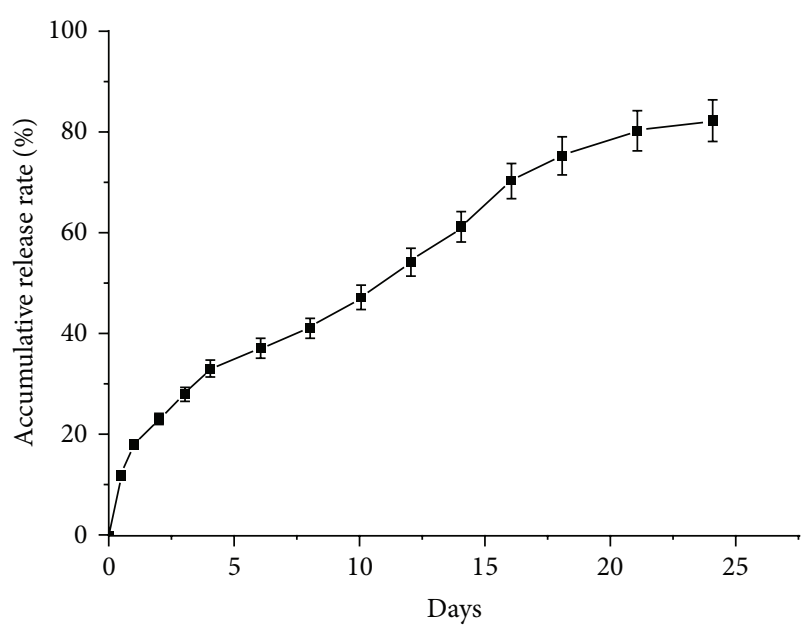

(b)

FIGURE 3: Changes of bFGF release rates from bFGF-loaded chitosan microspheres. Results are averages of three independent experiments. Data represent mean \pm SEM.

peptide delivery. This event causes undesirable loss and potentially toxic levels of therapeutic from the microsphere. Thus, modulation of release is of particular importance in these studies because little is known regarding the therapeutic dose of protein and/or peptide in humans [12, 17]. Burst release rate is an important indicator to assess microsphere drug delivery system, and the values are approximately 20 $30 \%$. In the present study, in vitro release process of the bFGFloaded chitosan microspheres showed relatively stable and smooth tread, and the cumulative burst release rate is $18.1 \%$ in the first day. The cumulative release rate is $82.1 \%$ from Day 2 to Day 24 , and the even value is approximately $53.30 \mathrm{ng} / \mathrm{mL}$ every two days. These results indicated in vitro release process of bFGF-loaded chitosan microspheres is slow; that is, the increased concentration of bFGF is lower every two days.

Schwann cells (SCs) are generated from neural crest cells and undergo extensive migration, proliferation, and maturation before they terminally differentiate. SCs are valid candidates for cell therapy of PNS injuries, being the glial as

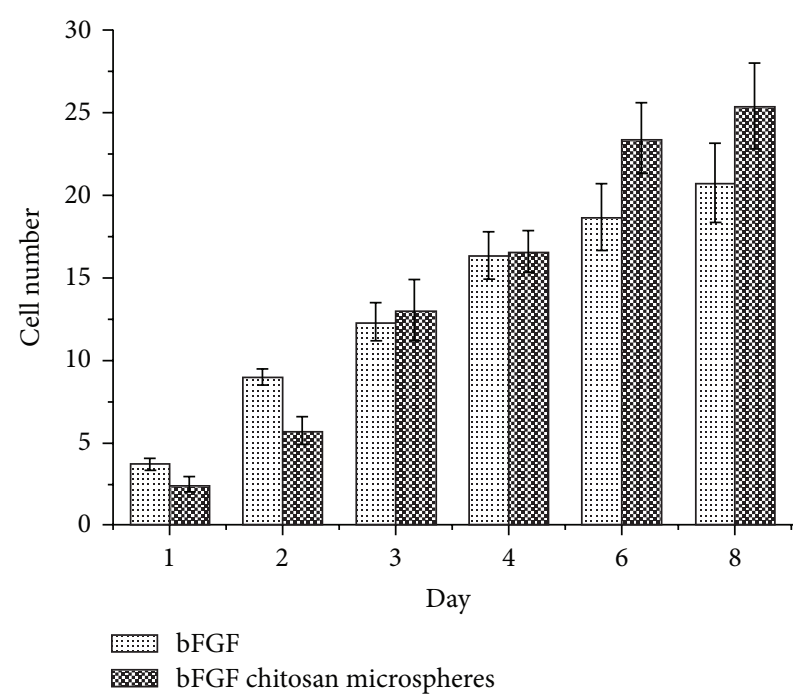

FIGURE 4: Effects of bFGF and bFGF-loaded chitosan microspheres on the cell number. Results are averages of three independent experiments. Data represent mean \pm SEM.

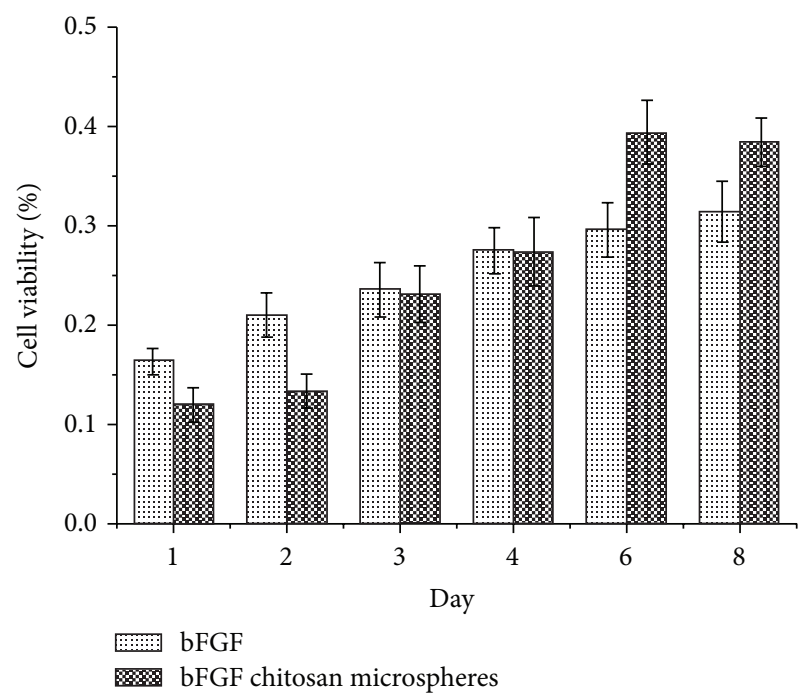

FIGURE 5: Effects of bFGF and bFGF-loaded chitosan microspheres on the cell viability. Results are averages of three independent experiments. Data represent mean \pm SEM.

well as myelinating cells in the PNS and playing a crucial role both in supporting peripheral axon regeneration and remyelination after injury $[18,19]$. It has been well established that artificial nerve grafts filled with naive SCs from neonatal stimulate peripheral nerve fiber regeneration. The growthpromoting effect is thought to occur as a result of the SCs ability to secrete a range of trophic factors including bFGF [20]. Wang et al. reported that the expression and secretion of sustained high levels of bFGF were observed in in vitro culture after exogenous bFGF mRNA modified Schwann cells. In addition, bFGF have mitogenic activity on the cultured Schwann cell. After bFGF binding to receptor of target cells, the transcription levels of ribosome gene will 


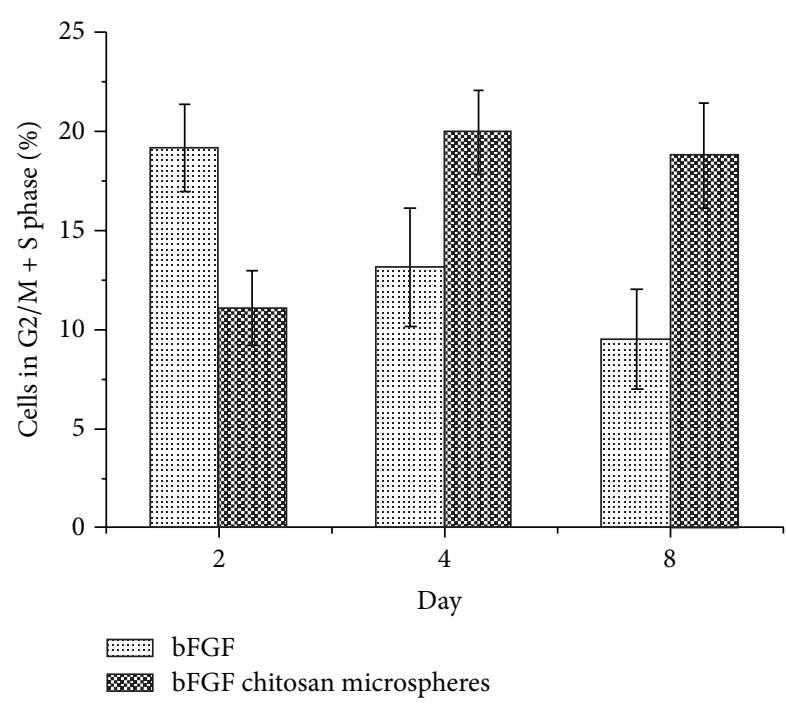

FIGURE 6: Effects of bFGF and bFGF-loaded chitosan microspheres on the cell phases. Results are averages of four independent experiments. Data represent mean \pm SEM.

accelerate by the influence of RNA polymerase I, and the cells were promoted $\mathrm{G}_{1}$-to-S phase and $\mathrm{G}_{1}$-to-S phase transition, which will shorten the $G_{1}$ phase and promote cell division and proliferation [21]. In the present study, results from cell number, cell viability, and cell phase suggested that the mitogenic activity in the bFGF-loaded chitosan microspheres groups is less than that in the bFGF groups at Days 1 and 2 , but the mitogenic activity in the bFGF-loaded chitosan microspheres groups is significantly higher than that in the bFGF groups at Days 6 and 8 (Figures 3, 4, and 5). In the bFGF and bFGF-loaded chitosan microspheres, the total amount of bFGF is the same. At Days 1 and 2, the bFGF concentration in the bFGF group is significantly higher than that in the bFGFloaded chitosan microspheres for sustained release process of microspheres groups. This may be consistent with the results of mitogenic activity. However, the bFGF concentration in the microspheres group gradually increased with the sustained release and increasing time, and the mitogenic activity in the microspheres group is significantly higher than that in the control groups at Days 6 and 8. These findings demonstrated that bFGF-loaded chitosan microspheres provide a suitable environment supporting the cellular structure, proliferation, and mineralization. However, the differentiation mechanism of Schwann cells by which the bFGF-loaded chitosan microspheres environment promoted cell proliferation is complex and needs further study.

\section{Conclusion}

In summary, a simple operation and emulsion crosslinking method for the preparation of bFGF-loaded chitosan microspheres has been developed, and the microspheres had smooth sphere surface, uniform distribution, and dispersion. Results of in vitro release showed that release rate is relatively stable and maintains the biological activity of bFGF. In addition, bFGF-loaded chitosan microspheres may be developed for the deployment of a controlled release growth factors delivery system to promote growth and mitogenic activity of cells. These findings suggest that bFGF-loaded chitosan microspheres are a promising biomaterial for improving skin regeneration efficacy and promoting vascularization.

\section{Conflict of Interests}

There is no conflict of interests to declare, and each author certifies that they have no commercial associations that might pose a conflict of interests in connection with this paper.

\section{References}

[1] C. Popovici, R. Roubin, F. Coulier, and D. Birnbaum, "An evolutionary history of the FGF superfamily," BioEssays, vol. 27, no. 8, pp. 849-857, 2005.

[2] N. Itoh and D. M. Ornitz, "Functional evolutionary history of the mouse Fgf gene family," Developmental Dynamics, vol. 237, no. 1 , pp. 18-27, 2008 .

[3] N. Itoh and D. M. Ornitz, "Fibroblast growth factors: from molecular evolution to roles in development, metabolism and disease," The Journal of Biochemistry, vol. 149, no. 2, pp. 121-130, 2011.

[4] G. Mudò, A. Bonomo, V. Di Liberto, M. Frinchi, K. Fuxe, and N. Belluardo, "The FGF-2/FGFRs neurotrophic system promotes neurogenesis in the adult brain," Journal of Neural Transmission, vol. 116, no. 8, pp. 995-1005, 2009.

[5] H. Maity, C. Karkaria, and J. Davagnino, "Effects of pH and arginine on the solubility and stability of a therapeutic protein (fibroblast growth factor 20): relationship between solubility and stability," Current Pharmaceutical Biotechnology, vol. 10, no. 6, pp. 609-625, 2009.

[6] K. Lee, E. A. Silva, and D. J. Mooney, "Growth factor deliverybased tissue engineering: general approaches and a review of recent developments," Journal of the Royal Society Interface, vol. 8, no. 55, pp. 153-170, 2011.

[7] R. Vasita and D. S. Katti, "Growth factor-delivery systems for tissue engineering: a materials perspective," Expert Review of Medical Devices, vol. 3, no. 1, pp. 29-47, 2006.

[8] E. Anitua, M. Sánchez, G. Orive, and I. Andia, "Delivering growth factors for therapeutics," Trends in Pharmacological Sciences, vol. 29, no. 1, pp. 37-41, 2008.

[9] Y. Shi and G. Huang, "Recent developments of biodegradable and biocompatible materials based micro/nanoparticles for delivering macromolecular therapeutics," Critical Reviews in Therapeutic Drug Carrier Systems, vol. 26, no. 1, pp. 29-84, 2009.

[10] S. Zhang and H. Uludağ, "Nanoparticulate systems for growth factor delivery," Pharmaceutical Research, vol. 26, no. 7, pp. 15611580, 2009.

[11] J. Varshosaz, "The promise of chitosan microspheres in drug delivery systems," Expert Opinion on Drug Delivery, vol. 4, no. 3, pp. 263-273, 2007.

[12] S. A. Agnihotri, N. N. Mallikarjuna, and T. M. Aminabhavi, "Recent advances on chitosan-based micro- and nanoparticles in drug delivery," Journal of Controlled Release, vol. 100, no. 1, pp. 5-28, 2004. 
[13] D. L. Nettles, S. H. Elder, and J. A. Gilbert, "Potential use of chitosan as a cell scaffold material for cartilage tissue engineering," Tissue Engineering, vol. 8, no. 6, pp. 1009-1016, 2002.

[14] M. Dash, F. Chiellini, R. M. Ottenbrite, and E. Chiellini, "Chitosan-a versatile semi-synthetic polymer in biomedical applications," Progress in Polymer Science, vol. 36, no. 8, pp. 9811014, 2011.

[15] X. Z. Shu and K. J. Zhu, "Controlled drug release properties of ionically cross-linked chitosan beads: the influence of anion structure," International Journal of Pharmaceutics, vol. 233, no. 1-2, pp. 217-225, 2002.

[16] C. Zeng, D. Cai, D. Quan et al., "Preparation of TGF- $\beta 1$ load chitosan microspheres and its properties in vitro," Journal of Sun Yat-Sen University (Medical sciences), vol. 26, no. 3, pp. 347-350, 2005.

[17] J. J. Wang, Z. W. Zeng, R. Z. Xiao et al., "Recent advances of chitosan nanoparticles as drug carriers," International Journal of Nanomedicine, vol. 6, pp. 765-774, 2011.

[18] K. Krick, M. Tammia, R. Martin, A. Höke, and H. Mao, "Signaling cue presentation and cell delivery to promote nerve regeneration," Current Opinion in Biotechnology, vol. 22, no. 5, pp. 741-746, 2011.

[19] H. C. Lehmann and A. Höke, "Schwann cells as a therapeutic target for peripheral neuropathies," CNS and Neurological Disorders-Drug Targets, vol. 9, no. 6, pp. 801-806, 2010.

[20] L. A. Pfister, M. Papaloïzos, H. P. Merkle, and B. Gander, "Nerve conduits and growth factor delivery in peripheral nerve repair," Journal of the Peripheral Nervous System, vol. 12, no. 2, pp. 6582, 2007.

[21] F. Wang, Z. Yuan, and W. Liu, "bFGF gene transfection and expression in Schwann cells," Chinese Journal of Bone and Joint Injury, vol. 24, no. 12, pp. 1089-1093, 2009. 

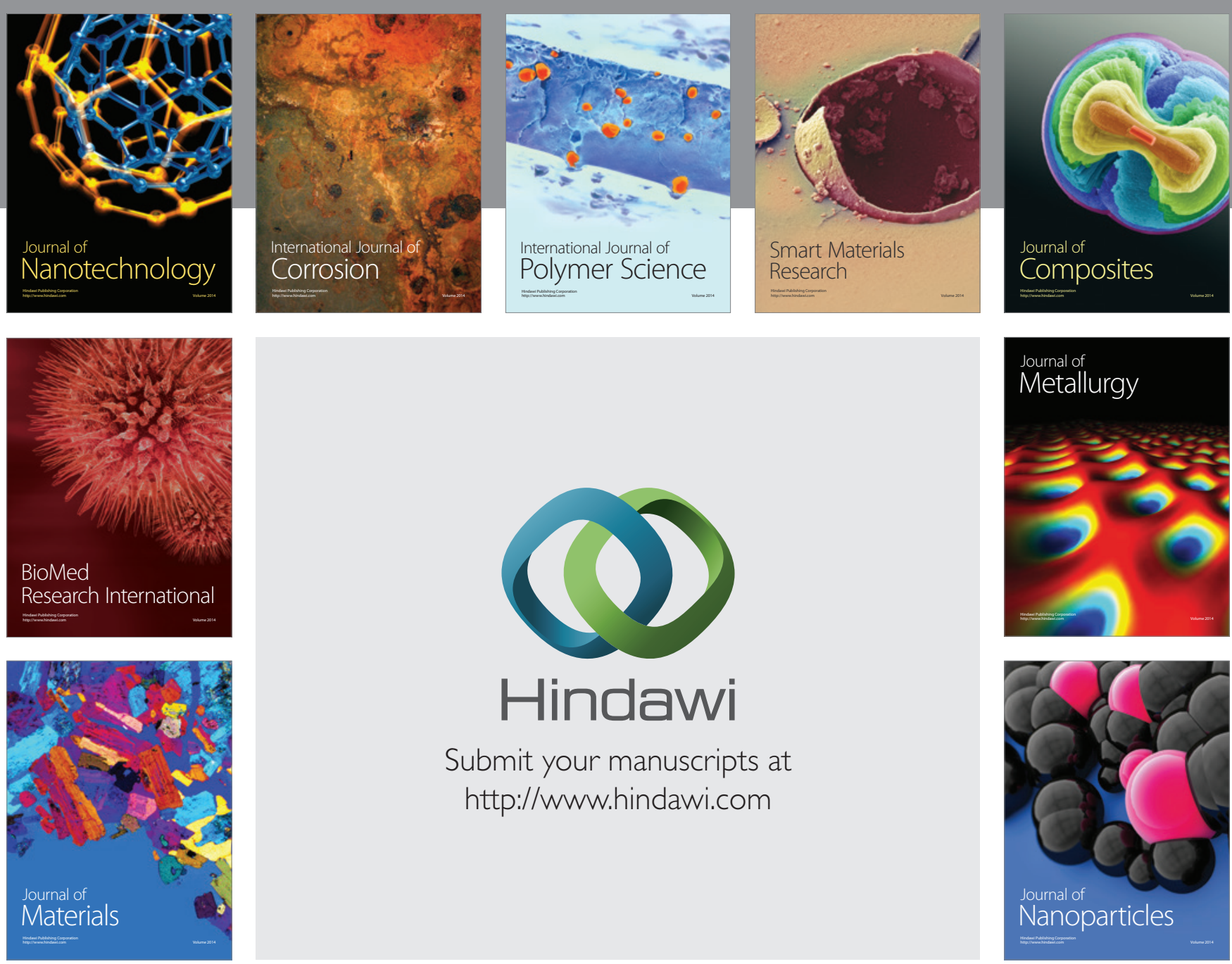

Submit your manuscripts at http://www.hindawi.com
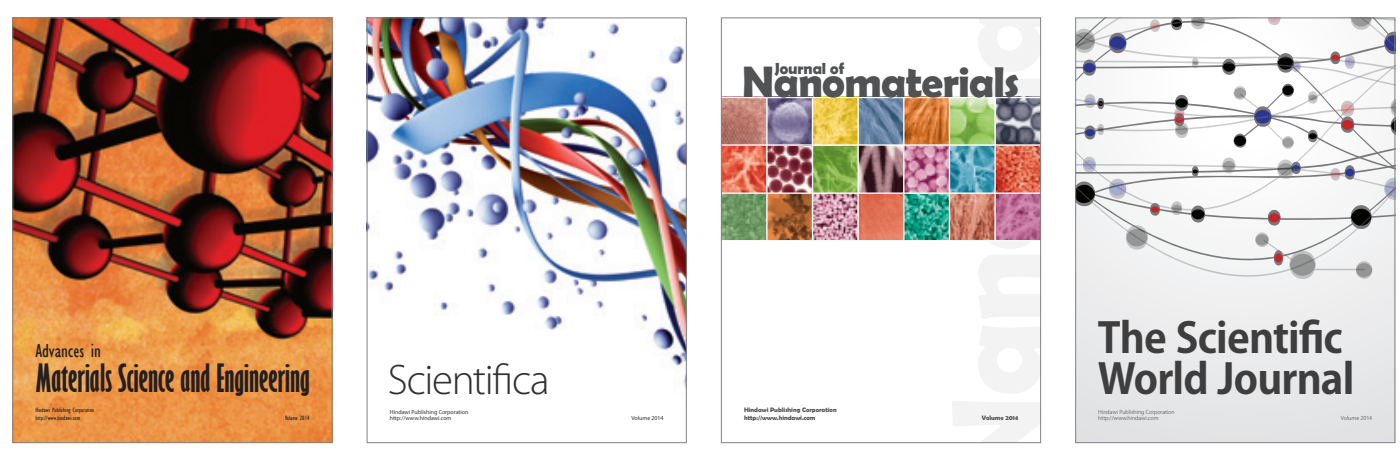

\section{The Scientific World Journal}
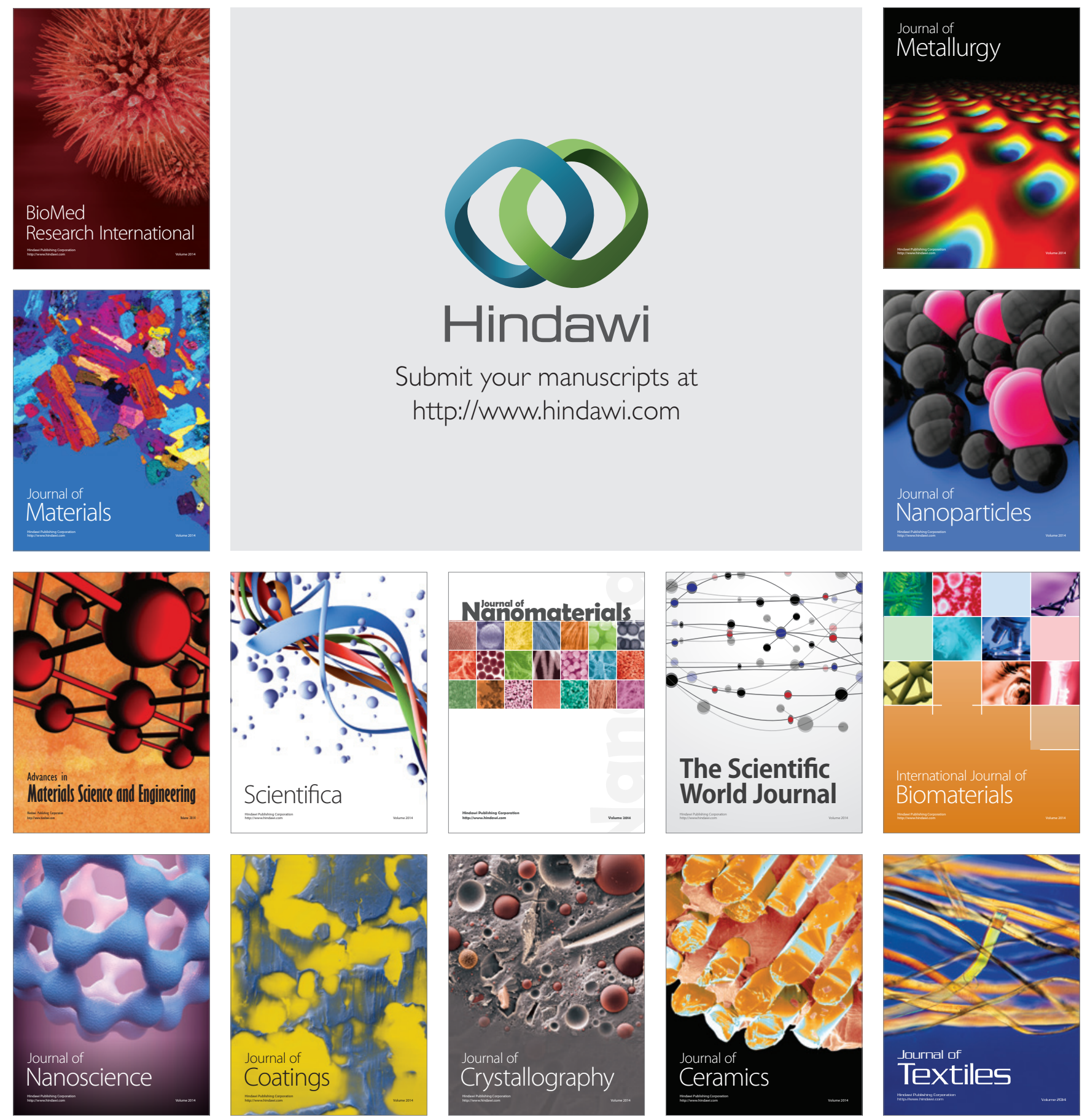